\title{
Proposal of an Enhanced Safety System on Board of the Inland Vessel
}

\author{
Martin Jurkovič ${ }^{1, *}$, Tomáš Kalina ${ }^{1}$, Robert Turčan ${ }^{1}$, and Bruno Gardlo ${ }^{2}$ \\ ${ }^{1}$ University of Žilina, Faculty of Operation and Economics of Transport and Communications, \\ Department of Water Transport, Univerzitná 1, Žilina 010 26, Slovak Republic \\ ${ }^{2}$ AIT Austrian Institute of Technology GmbH, Center for Technology Experience, Giefinggasse 2, \\ 1210 Wien, Austria
}

\begin{abstract}
The share of human factor in the occurrence of navigation accidents represents a high proportion of the total accident rate. Elimination of the human factor responsible for the occurrence of navigational accidents would contribute to the overall safety of navigation on inland waterways. The main causes of human-induced navigation accidents include inattention, fatigue or poor concentration on vessel control. The design of the system for increasing navigation safety consists of a system for monitoring the life functions of a crew member as well as a position of the vessel towards the fairway / shore. The first level focuses on tracking the life function of the vessel's crew / boat master. The second level addresses the position of the vessel and the possibility of increasing safety by cooperating with the data provided by Automatic Identification System - AIS. The proposal of the system represents interconnection of both levels and brings high degree of control of the vessel in extraordinary situations.
\end{abstract}

\section{Introduction}

The system of monitoring and recording important life functions or parameters of the driver's state of consciousness is not new in the automotive industry. Volvo has been working on such a system since 2005 , but it worked on the principle of tracking secondary data, such as directional control, braking frequency, involuntary lane departure, etc. The control unit with a predetermined computational algorithm then evaluated the nonstandard behavior based on the data obtained. At present, these systems are even more refined $[1,2]$.

A new generation of systems should bring direct driver monitoring while driving, based on the sensors built into the dashboard. The sensor monitors the direction in which the driver looks ahead, how far he has open his eyes or in which position is his head. From the scanned data, it is subsequently possible to evaluate whether the driver is tired, asleep or disintegrating. In the case of a negative result, the software will automatically ensure that the vehicle does not break out of the lane or collide with another vehicle [3].

Due to the impact of the human factor in navigational accidents, similar systems should be installed on inland waterway vessels as well. Currently inland waterway vessels are not

* Corresponding author: martin.jurkovic@fpedas.uniza.sk 
equipped with an early warning system or collision tracking system. Implementing at least one of the system could reduce the number of human-induced accidents.

\section{Methodology of system operation}

The system of monitoring and evaluating important life functions is a complex and detailed processing of the obtained data, therefore it is suitable for use on inland waterway vessels. It would consist of several sensors divided into two levels of monitoring. For each level, the following parameters would be monitored:

\section{First level:}

- eye movement - percentage of eye lid closure and tracking of the eye,

- Brain activity (diffuse optical tomography - DOT method),

- breathing and heart activity (Emotional Intelligence EQ - Radio Method).

\section{Second level:}

- the relative position of the vessel against the fairway (AIS system required),

- slowdown and acceleration of the vessel (AIS required).

Both algorithms would be an integral part of a complex system where the software would collect, monitor, and evaluate information coming from subsystems at a lower rating level. Based on the outcome of the assessment, the system would take all necessary measures to reduce or eliminate the potential hazard. The co-operation of each level is shown in Fig. $1[4,5]$.

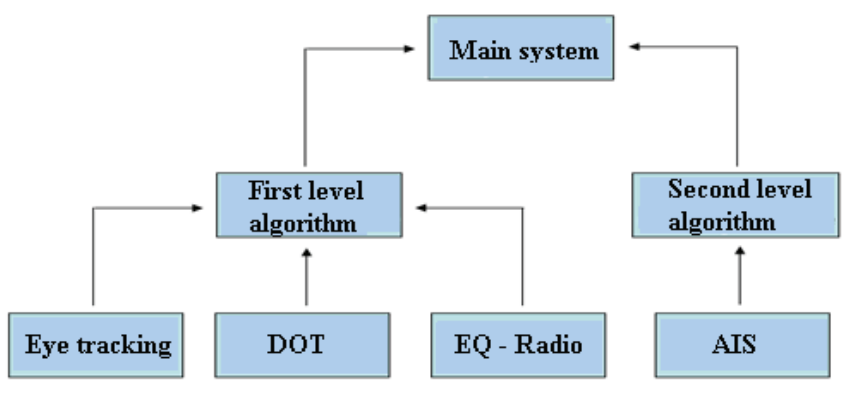

Fig. 1. Scheme of the monitoring system. Source: authors

\subsection{Creating a network of sensors}

The measurement of selected parameters would be ensured by the network of specific sensors. After collecting enough data, the data would be sent through CAN or "Controller Area Network" for evaluation to the control unit. CAN is a standard communication system used in automobiles to interconnect components such as an engine, transmission, chassis or brakes. CAN is a bus that is used for internal communication between sensors and functional units. It is used mainly in the automotive diagnostic system or in the industrial sphere for machinery and lines [6]. The network would consist of one complex or more specific CAN systems for each parameter.

\subsection{Evaluation of obtained data}

The evaluation would consist of two separate assessment algorithms, each for one level of tracking. In the system, both algorithms would be interconnected and co-operated. Their interaction is illustrated in Fig. 2. 


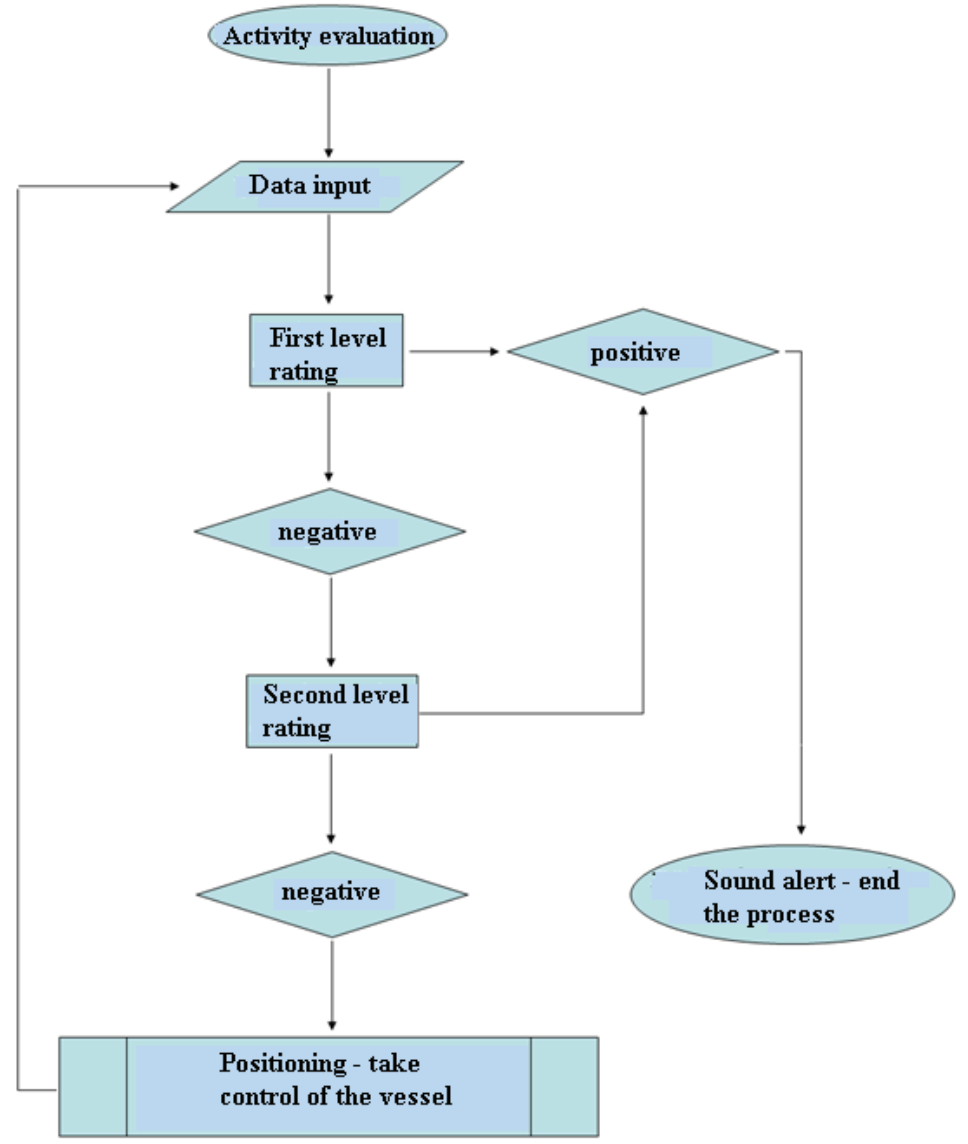

Fig. 2. Interaction of algorithms. Source: authors

\section{Components of the monitoring system}

The monitoring system would collect, monitor and evaluate information from individual subsystems. Based on its importance, the most appropriate measure will be adopted in terms of navigation and crew safety.

\subsection{Eye movement - percentage of eye lid closure and tracking of the eye}

Eye movement, more accurate motion and percentage closure of the eyelid, would capture the camera system installed on the bridge. Motion capture would be used by cameras with a certain scanning frequency. Due to the different velocity of eyelid movement during glans and fatigue, high-frequency scanning is required. For this reason, installation of a highfrequency camera is appropriate [7]. The algorithm of the system would work on the principle of recognizing the facial skin color as follows:

- recognition of skin and non-skinned pixels (facial and non-face recognition),

- rendering of the face part,

- pixel layout into multiple areas (definition of face and non-face areas).

Through a binary pixel set, the point that belongs to the face area is assigned a value of 1. The point that does not belong to the face area is assigned a value of 0 . Based on the 
face's conversion to a black and white image, the outline of the shape, eyebrow, eyes, and part of the nose is drawn. The algorithm then calculates the coordinates of the eye according to the part of the eye (the eyebrow and the lower lid of the eye). Once the position of both eyes is detected, the system can record the intensity of the change from the closed to the open eyelid [7].

\subsection{Monitoring of the brain activity}

Monitoring of brain activity and measurement of individual essential functions would be performed using a relatively new, non-invasive brain scan method - DOT method. The method uses brain scan using LEDs. The results are comparable to magnetic resonance [8].

The method is referred to diffusion optical tomography and shows processes that are running at various levels of the brain. It works on the principle of transmitting light to the cerebral cortex. The light is then captured backwards. This process is followed by dynamic changes in the color of the tissue in the brain. Coloration occurs in ascending or declining cerebral activity, when neuronal activity increases in the brain.

To ensure the functionality of the system on the vessel (bridge), the following components are required:

- covering the entire bridge with LEDs,

- software for recording and evaluating brain activity.

\subsection{Breathing and hearth activity}

The analysis of breathing and cardiac activities without audio-visual or physiological data coming from the sensors on the human body is possible using EQ - Radio technology. The technology was developed and completed in 2016 at the Massachusetts Institute of Technology. It works on the principle of transmitting, reflecting and receiving a wireless signal to the human organism. The accuracy of heart rate measurement is the same as for the ECG. The error of measurement is only 0.3 percent [9].

The software processes the received data based on the selection of breathing rhythm and cardiac activity. When breathing, the system records a change in breathing rate (number of breaths and breaths over the reference period). Heart activity is evaluated based on a change in the number of heart strokes over the time. This can lead to the discovery of emotion as fear, excitement, disgust. The intensity of heart activity is directly proportional to fatigue. When falling asleep, heart activity decreases.

Analyzing and evaluating of the cardiac activity and respiration would consist of:

- positive / negative data evaluation;

- in the case of a positive evaluation, the whole cycle would be repeated, without warning,

- in case of a negative evaluation, the software will send the data to the control unit the system will take all the necessary measures to eliminate the hazard.

\subsection{The relative position of the vessel to fairway}

The most optimal solution for tracking the vessel's relative position towards the fairway is the AIS (Automatic Identification System). AIS is used not only for inland navigation. AIS stores and displays position, speed, rate and vessel information (name, number, calling sign, vessel type). The system works on the principle of information exchange between coastal stations or directly between vessels $[10,11]$.

Vessels equipped with standard AIS stations receive and transmit data automatically at specified intervals. The advantage is cooperation (refill) with radar due to different 
characteristics of both systems. For integration into the second stage of the monitoring system, following information is needed:

- the precise position of the vessel,

- course and speed of the vessel.

The communication is via a digitized radio station, which can be complemented by a data transfer modulation device. Simple design is an advantage in terms of interconnection, since there is no need to install any additional elements into the vessel. The system would only be complemented by software for recording, monitoring and evaluating the optimal position of the vessel on the voyage. If the software evaluates that the position of the vessel to the voyage is not optimal, it would send the information to the monitoring system. The second evaluation algorithm would then alert the boat master [12].

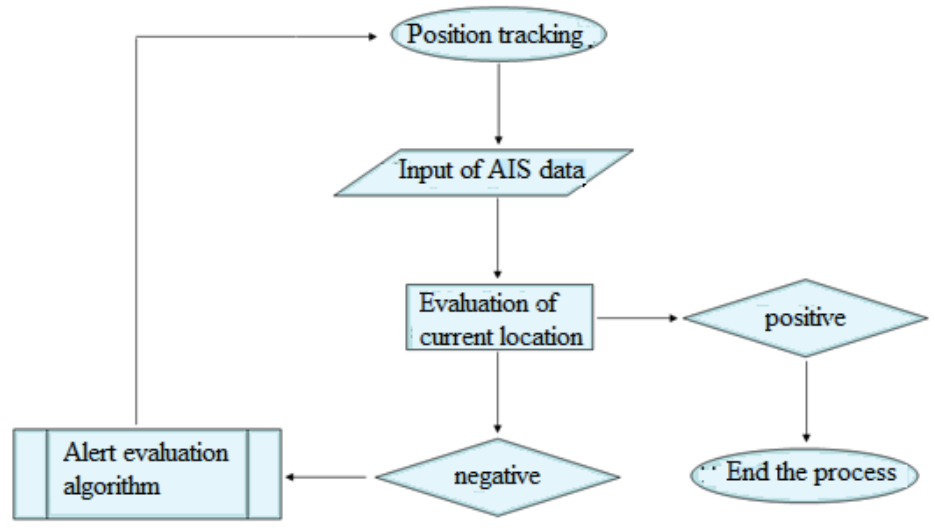

Fig. 3. Methodology of vessel tracking process in the voyage. Source: authors

An essential element in position tracking in combination with the evaluation algorithm is to determine the permissible deviation, since in the case of dynamic changes on the waterway it is not always possible to maintain the optimal system rate. For downstream and upstream navigation, it is necessary to specify a specific deviation for each type of voyage.

\subsection{Acceleration and deceleration of the vessel}

AIS can be used again to monitor vessel speed. The system provides information about the vessel speed based on satellite tracking. The system would have integrated software with its own evaluation algorithm. The software would process and evaluate critical vessel speed data. Depending on the negative or positive result, it would take appropriate measures as was mentioned above. In the case of a positive evaluation, the entire process starts from the beginning. On the other hand, in the case of a negative evaluation, the system would highlight a potential hazard.

A principal element of the speed tracking system is to determine the optimal speed of the vessel for individual sections of the waterway. The cooperation with the electronic map system is necessary, although speed monitoring is not necessary in all river sections. Therefore, only sections with increased risk of accidents would be monitored. Mainly:

- places with fords, shallows or straits,

- places with increased vessel density,

- anchorages and turning positions,

- port entrances, lock chambers,

- entrances to navigable bridges and tunnels, 
- places where navigation is restricted.

\section{Conclusion}

At present, there is no system of early warning or monitoring of crew safety on inland vessels. The introduction of such system could significantly reduce accidents and related negative impacts. The design of the monitoring system consists of two levels. The first is to monitor the life function of the vessel's crew on the bridge. The latter tracks the optimal route, position and speed of the vessel in the voyage. Their interconnection and cooperation would ensure a high degree of control of the vessel in extraordinary situations. Modern, non-invasive systems have been selected to monitor life functions.

DOT Brain Measurement System or EQ - Radio System for Measurement of Heart and Breast Activity. Optimal tracking and location of the vessel would be ensured by AIS enhanced with software dedicated to evaluating the optimal and current position and speed of the vessel. It is a question of implementing such a system on vessels. New breakthrough technologies usually bring with them a huge cost of research and development that may be an obstacle to the implementation of such a project.

This paper is supported by the research project "From horse-drawn railway to intermodal transport" within Visegrad Fund.

\section{References}

1. R. Kampf, O. Stopka, L. Bartuska, K. Zeman, Transport Means - Proceedings of the 19th International Scientific Conference on Transport Means, 143-146 (Kaunas, Lithuania: Kaunas University of Technology, 2015)

2. M. Mikusova, J. Gnap, CIT 2016 (Valencia, Spain, 2016)

3. T. Andrejcak, Pravda 7 (2014)

4. I. Kubasakova, R. Kampf, O. Stopka, Communications: scientific letters of the University of Žilina 16, 2, 9-13 (2014)

5. J. Gasparik, V. Luptak, P. Mesko, ICTTE - International conference on Traffic and transport engineering, 388-392 (Belgrade, Serbia, 2016)

6. CAN system, A Serial Bus System, Available online: http://www.esd-electronicsusa.com/Controller-Area-Network-CAN-Introduction.html (2016)

7. J. Rakus, (STU Faculty of Electrical Engineering and Informatics, Bratislava, Slovak Republic, 2011)

8. A. Eggebrecht, Available online: https://www.ncbi.nlm.nih.gov/pubmed/25595183 (2016)

9. A. Conner, Simons, R. Gordon, MIT, 6-8 (Cambridge, USA, 2016)

10. RIS, General Information, Available online: http://www.ris.eu/general/what_is_ris_/ais (2009)

11. T. Skrucany, J. Gnap, Applied Mechanics and Materials 617, 296-301 (2014)

12. J. Zamecnik, J. Jagelcak, J. Sosedova, LOGI 2015, 97-105 (České Budějovice, Czech Republic, 2015) 